\title{
Stochastic Averaging Using Elliptic Functions to Study Nonlinear Stochastic Systems
}

\author{
WIN-MIN TIEN and N. SRI NAMACHCHIVAYA \\ Aeronautical and Astronautical Engineering Department, University of Illinois at Urbana-Champaign, Urbana, IL 61801, \\ U.S.A. \\ V. T. COPPOLA \\ Department of Aerospace Engineering, University of Michigan, Ann Arbor, MI 48109, U.S.A.
}

(Received: 13 September 1991; accepted: 11 September 1992)

\begin{abstract}
In this paper, a new scheme of stochastic averaging using elliptic functions is presented that approximates nonlinear dynamical systems with strong cubic nonlinearities in the presence of noise by a set of Itô differential equations. This is an extension of some recent results presented in deterministic dynamical systems. The second order nonlinear differential equation that is examined in this work can be expressed as $\ddot{x}+c_{1} x+c_{3} x^{3}+\varepsilon f(x, \dot{x})+\varepsilon^{1 / 2} g(x, \dot{x}, \xi(t))=0$, where $c_{1}$ and $c_{3}$ are given constants, $\xi(t)$ is stationary stochastic process with zero mean and $\varepsilon \ll 1$ is a small parameter. This method involves the laborious manipulation of Jacobian elliptic functions such as $c n, d n$ and $s n$ rather than the usual trigonometric functions. The use of a symbolic language such as Mathematica reduces the computational effort and allows us to express the results in a convenient form. The resulting equations are Markov approximations of amplitude and phase involving integrals of elliptic functions. Finally, this method was applied to study some standard second order systems.
\end{abstract}

Key words: Stochastic averaging, nonlinear systems, elliptic functions, probability density.

\section{Introduction}

The method of stochastic averaging involves the convergence of a parametrized sequence $\left\{x^{e}(t)\right\}$ of processes to a limit process in some specific sense. Consider a physical model $\dot{x}^{\varepsilon}(t)=$ $b^{\varepsilon}\left(x^{\varepsilon}(t)\right)+\sigma^{\varepsilon}\left(x^{\varepsilon}(t)\right) \xi^{\varepsilon}(t)$ defined in a suitable parametrized family; the averaging method, then, finds a process $x^{\prime \prime}(t)$ such that $x^{\varepsilon}(t) \rightarrow x^{0}(t)$ in some specific sense as $\varepsilon \rightarrow 0$. It is important that the limit process $x^{0}(t)$ obtained by this procedure be much more tractable mathematically than the true physical process, and the parameter value $\varepsilon$, corresponding to the physical process, be small enough to yield a good approximation. The classical stochastic averaging method, which was established by Stratonovich [15] by a physically rigorous method, has been widely used to approximate physical processes with certain smoothness and differentiability conditions by a diffusive Markov process. A mathematically rigorous proof of this result was given by Khasminskii [6]. The applicability of this method to engineering problems is obvious from the overwhelming number of papers in the literature, notably, Ariaratnam and Srikantaiah [1], Roberts and Spanos [11] and Sri Namachchivaya and Ariaratnam [13]. A physical interpretation of this method which is more appealing to engineers is given by Lin [8]. This method provides Markov approximation solutions to quasi-linear differential equations of the form

$$
\ddot{x}+\omega^{2} x+\varepsilon f(x, \dot{x})+\varepsilon^{1 / 2} g(x, \dot{x}, \xi(t))=0
$$


where $\omega$ is system frequency and $\xi(t)$ represents a parametric random excitation. However, there are some disadvantages to this method as we shall point out in the following examples.

The first example is the Duffing's equation with parametric random excitation given by

$$
\ddot{x}+\omega^{2} x+\varepsilon\left(\alpha x^{3}+\rho \dot{x}\right)+\varepsilon^{1 / 2} x \xi(t)=0 .
$$

The solution of the unperturbed equation can be written as $x=A \cos (\omega t+\phi)$, where $A$ and $\phi$ represent the amplitude and phase of the excitation, respectively. One of the motivations for the use of averaging is to obtain an amplitude equation which is independent of phase, so that the response of equation (2) is studied using the amplitude equation alone. Thus, by using the classical stochastic averaging method, the Itô equations of $A$ and $\phi$ are obtained as

$$
\begin{aligned}
& \mathrm{d} A=\varepsilon\left[\frac{-\rho A}{2}+\frac{3 A}{16 \omega^{2}} S_{\xi \xi}(2 \omega)\right] \mathrm{d} t+\varepsilon^{1 / 2}\left[\frac{A^{2} S_{\xi \xi}(2 \omega)}{8 \omega^{2}}\right]^{1 / 2} \mathrm{~d} w \\
& \mathrm{~d} \phi=\varepsilon\left[\frac{3 \alpha A^{2}}{8 \omega}+\frac{1}{8 \omega^{2}} \Psi_{\xi \xi}(2 \omega)\right] \mathrm{d} t+\varepsilon^{1 / 2}\left[\frac{P c(0)}{4 \omega^{2}}+\frac{S_{\xi \xi}(2 \omega)}{8 \omega^{2}}\right]^{1 / 2} \mathrm{~d} w
\end{aligned}
$$

where $\mathrm{d} w$ is the Wiener process and

$$
S_{\xi \xi}(\cdot)=2 \int_{0}^{\infty} R_{\xi \xi}(\tau) \cos (\cdot) \tau \mathrm{d} \tau, \quad \psi_{\xi \xi}(\cdot)=2 \int_{0}^{\infty} R_{\xi \xi}(\tau) \sin (\cdot) \tau \mathrm{d} \tau
$$

$R_{\xi \xi}(\tau)$ is defined as $E[\xi(t) \xi(t+\tau)]$. From equations (3) and (4) we observe that the cubic nonlinearity appears only in the phase equation. Furthermore, the amplitude equation contains only the linear expression. Thus, the effect of nonlinearity in equation (2) is eliminated in the averaging procedure yielding only the linear response.

As a second example we consider the Duffing-van der Pol equation which represents a large amount of nonlinear phenomena

$$
\ddot{x}+\omega^{2} x+\varepsilon\left(\alpha x^{3}+\delta x^{2} \dot{x}\right)+\varepsilon^{1 / 2} x \xi(t)=0 .
$$

As in the previous example, the Itô equations of $A$ and $\phi$ are

$$
\begin{aligned}
& \mathrm{d} A=\varepsilon\left[\frac{-\delta A^{3}}{8}+\frac{3 A}{16 \omega^{2}} S_{\xi \xi}(2 \omega)\right] \mathrm{d} t+\varepsilon^{1 / 2}\left[\frac{A^{2} S_{\xi \xi}(2 \omega)}{8 \omega^{2}}\right]^{1 / 2} \mathrm{~d} w, \\
& \mathrm{~d} A=\varepsilon\left[\frac{-\delta A^{3}}{8}+\frac{3 A}{16 \omega^{2}} S_{\xi \xi}(2 \omega)\right] \mathrm{d} t+\varepsilon^{1 / 2}\left[\frac{A^{2} S_{\xi \xi}(2 \omega)}{8 \omega^{2}}\right]^{1 / 2} \mathrm{~d} w .
\end{aligned}
$$

Although nonlinear damping in this example appears in the amplitude equation (6), the cubic nonlinearity still has no influence on this equation and only appears in the phase equation. It is worth mentioning that, in the past, only damping type nonlinearities were considered while using the method of averaging for second order systems.

From the examples given above, it is necessary for us to develop a new scheme to overcome the disadvantage of the classical method. A general procedure will be to accommodate all strong potential nonlinearities. In this paper, however, due to the tedious algebra for the general cases, 
we shall extend the classical stochastic averaging method to the case of stochastically excited systems with strong cubic nonlinearity, i.e.,

$$
\ddot{x}+c_{1} x+c_{3} x^{3}+\varepsilon f(x, \dot{x})+\varepsilon^{1 / 2} g(x, \dot{x}, \xi(t))=0
$$

where $c_{1}$ and $c_{3}$ are constants, $\varepsilon$ is a small constant coefficient, and $\xi(t)$ represents a real valued zero mean stochastic process with autocorrelations that go to zero rapidly for large times, i.e., it satisfies a strong mixing condition

$$
\int_{0}^{\infty} \tau^{m} R_{\xi \xi}(\tau) \mathrm{d} \tau<\infty, \quad m=0,1,2 .
$$

The above condition implies that the power spectral density of the $\xi(t)$ processes, and their first two derivatives are bounded at zero frequency. With the assumption given above, the model can be treated using the results of stochastic averaging. In this paper, we extend the results of Coppola and Rand [4] and Coppola [5] for nonlinear second order deterministic system to include stochastic excitations. The Markov approximation of this dynamical system will be used to study the nonlinear behavior by obtaining the stationary probability density function and moment response. It is worth mentioning that the second order system similar to equation (8) has been studied by Roberts [12] and Sri Namachchivaya [14] using energy envelope formulation. However, the methodology presented there is quite different from those reported by previous results.

\section{Variation of Parameters}

In order to obtain a set of equations in "standard form" necessary for the method of averaging, we first consider the unperturbed system of the form

$$
\ddot{x}+c_{1} x+c_{3} x^{3}=0
$$

Equation (9) possesses a general solution of the form

$$
\begin{aligned}
& x=A c n(u, k)=A c n(\omega t+\varphi, k) \\
& \dot{x}=A \omega \frac{\partial c n(u, k)}{\partial u}
\end{aligned}
$$

with

$$
\omega^{2}=c_{1}+c_{3} A^{2}, \quad k^{2}=\frac{c_{3} A^{2}}{2\left(c_{1}+c_{3} A^{2}\right)}
$$

where amplitude $A$ and frequency $\omega$ are positive constants. The $c n(u, k)$ function represents a Jacobian elliptic function which is a periodic function depending on both its argument $u=\omega t+\varphi$ and its modulus $k=k(A)$. In this paper, all formulas and conventions concerning Jacobian elliptic functions are taken from Byrd and Friedman [3]. Furthermore, the $k$ and $\omega$ are known in terms of $A$. Hence, the amplitude $A$ and the phase angle $\varphi$ are two arbitrary constants determined by the initial conditions. 
In order to obtain a solution to equation (8) when $\varepsilon \neq 0$, we use the variation of parameters method so that $A=A(t)$ and $\varphi=\varphi(t)$. The methodology presented herein is similar to that given by Coppola and Rand [4]. Differentiating (10a), we obtain

$$
\begin{aligned}
\dot{x} & =\dot{A} c n+A \frac{\partial c n}{\partial u} \frac{\mathrm{d} u}{\mathrm{~d} t}+A \frac{\partial c n}{\partial k} \frac{\mathrm{d} k}{\mathrm{~d} t} \\
& =\omega A c n^{\prime}+\dot{A} c n+A c n^{\prime}\left(\dot{\omega} t+\dot{\varphi}-\frac{\delta u}{\delta k} \dot{k}\right)
\end{aligned}
$$

where $\partial c n / \partial k=-c n^{\prime} \delta u / \delta k$ and

$$
\delta u / \delta k=u / K \mathrm{~d} K / \mathrm{d} k+z(u) /\left(k\left(1-k^{2}\right)\right)-k /\left(1-k^{2}\right) s n c n / d n
$$

$K \equiv K(k)$ denotes the complete elliptic integral of the first kind, $z(u)$ denotes the Jacobian Zeta function $(\cdot)^{\prime}$ denotes $\partial(\cdot) / \partial u$ [e.g., $\left.c n^{\prime}=\partial c n / \partial u=-d n s n\right]$, and the arguments $(u, k)$ of the Jacobian elliptic functions have been suppressed. The notation $\delta u / \delta k$ has been used instead of $\mathrm{d} u / \mathrm{d} k$ in Coppola $[4,5]$ to emphasize that the expression is not a total derivative. These notations will be used throughout this paper. By using equation (10c), we find

$$
\dot{\omega}=\frac{c_{3} A}{\omega} \dot{A} \quad \dot{k}=2 \frac{c_{1}}{c_{3}} \frac{k^{3}}{A^{3}} \dot{A}
$$

Substituting equations (12) into equation (11), one obtains

$$
\dot{x}=\omega A c n^{\prime}+A\left(c n+\frac{c_{3} A^{2}}{\omega} t c n^{\prime}-2 \frac{c_{1}}{c_{3}} \frac{k^{3}}{A^{2}} \frac{\delta u}{\delta k} c n^{\prime}\right)+A c n^{\prime} \dot{\phi} .
$$

Differentiating $\dot{x}$ in equation (10b), we obtain

$$
\begin{aligned}
\ddot{x}= & \omega^{2} A c n^{\prime \prime}+A \omega c n^{\prime \prime} \dot{\varphi}+\dot{A}\left[\omega c n^{\prime}+2 k^{2} \omega c n^{\prime}+\frac{c_{1} k^{2}}{\omega} \frac{s n^{3}}{d n}\right. \\
& \left.+c n^{\prime \prime} c_{2} A^{2} t-c n^{\prime \prime} \frac{c_{1} k^{2}}{\omega} \frac{\delta u}{\delta k}\right] .
\end{aligned}
$$

Comparing equations (13) and (10b) yields

$$
\dot{A}\left(c n+\frac{c_{3} A^{2}}{\omega} t c n^{\prime}-\frac{c_{1}}{\omega^{2}} k \frac{\delta u}{\delta k} c n^{\prime}\right)+A c n^{\prime} \dot{\varphi}=0 .
$$

From equations (14) and (8), another restricted equation can be written as

$$
\begin{aligned}
& A \omega c n^{\prime \prime} \dot{\varphi}+\dot{A}\left[\left(2 k^{2} \omega+\omega\right) c n^{\prime}+\frac{c_{1}}{\omega} k^{2} \frac{s n^{3}}{d n}+c n^{\prime \prime}\left(c_{3} A^{2} t-\frac{c_{1}}{\omega} k \frac{\delta u}{\delta k}\right)\right] \\
& +\varepsilon f\left(A c n, A \omega c n^{\prime}\right)+\varepsilon^{1 / 2} g\left(A c n, A \omega c n^{\prime}, \xi(t)\right)=0 .
\end{aligned}
$$

Equations (15) and (16) are solved for $\dot{A}$ and $\dot{\varphi}$. Substituting $\dot{\varphi}$ from equation (15) into equation 
(16), we obtain

$$
\dot{A}\left[-\omega c n^{\prime \prime} c n+\left(2 k^{2}+1\right) \omega\left(c n^{\prime}\right)^{2}-\left(1-2 k^{2}\right) k^{2} \omega s n^{4}\right]+\varepsilon f+\varepsilon^{1 / 2} g=0 .
$$

After some simplification, the slowly varying amplitude equation of order $\varepsilon$ can be obtained as

$$
\dot{A}=\frac{-1}{\omega}\left(\varepsilon f c n^{\prime}+\varepsilon^{1 / 2} g c n^{\prime}\right) \text {. }
$$

Due to the non-periodicity for the equation of $\dot{\varphi}$ derived from equation (10), the $\dot{\varphi}$ equation is not suitable for the averaging procedure. A new angle variable $\phi$ is chosen such that the variational equation will be periodic. To this end, let $u=\omega t+\varphi=4 K \phi, \dot{\phi}$ can be derived as

$$
\dot{\phi}=\frac{\omega}{4 K}+\frac{\dot{\omega} t}{4 K}+\frac{\dot{\varphi}}{4 K}-\frac{u}{4 K^{2}} \frac{\mathrm{d} K}{\mathrm{~d} k} \dot{k}
$$

By substituting equations (14) and (17) into the above equation, we obtain

$$
\dot{\phi}=\frac{\omega}{4 K}+\frac{1}{4 K A \omega}\left(\varepsilon f+\varepsilon^{1 / 2} g\right)\left[c n-\frac{\left(1-2 k^{2}\right)}{\left(1-k^{2}\right)}\left(z(u) c n^{\prime}+k^{2}\left(1-c n^{2}\right) c n\right)\right] .
$$

Now the amplitude equation and phase angle equation are in the "standard form" and ready for averaging. In order to simplify the notation we rewrite equations (17) and (19) as

$$
\begin{aligned}
& \dot{A}=\varepsilon F_{1}(A, \phi)+\varepsilon^{1 / 2} G_{1}(A, \phi, \xi(t)) \\
& \dot{\phi}=\Omega(A)+\varepsilon F_{2}(A, \phi)+\varepsilon^{1 / 2} G_{2}(A, \phi, \xi(t))
\end{aligned}
$$

where

$$
\begin{aligned}
& F_{1}=\frac{-1}{\omega} f c n^{\prime} \quad G_{1}=\frac{-1}{\omega} g c n^{\prime} \\
& \Omega(A)=\frac{\omega}{4 K} \\
& F_{2}=\frac{f}{4 K A \omega}\left[c n-\frac{\left(1-2 k^{2}\right)}{\left(1-k^{2}\right)}\left(z(u) c n^{\prime}+k^{2}\left(1-c n^{2}\right) c n\right)\right] \\
& G_{2}=\frac{g}{4 K A \omega}\left[c n-\frac{\left(1-2 k^{2}\right)}{\left(1-k^{2}\right)}\left(z(u) c n^{\prime}+k^{2}\left(1-c n^{2}\right) c n\right)\right] .
\end{aligned}
$$

The Jacobian elliptic functions are dependent on the independent variables $(A, \phi)$ and their differentiation with respect to $A$ and $\phi$ are periodic, for instance,

$$
\begin{aligned}
& \frac{\partial c n}{\partial A}=\left(z s n d n-k^{2} c n\left(1-c n^{2}\right)\right) \frac{c_{1}}{k^{\prime} A \omega^{2}} \\
& \frac{\partial c n}{\partial \phi}=-4 K d n s n .
\end{aligned}
$$




\section{Method of Stochastic Averaging}

The stochastic averaging procedure was proposed by Stratonovich [15] and later justified rigorously by Khasminskii [6]. Since then, several authors have expanded on this theory. Papanicolaou and Borodin [2] provided the same results under essentially less stringent assumptions. Furthermore, the application of stochastic averaging theory to singular-perturbation problem was given by Khasminskii [7] and Papanicolaou et al. [10]. Let's consider the process $x_{t}^{E}$ of the following equation

$$
\dot{x}=\varepsilon^{2} F^{0}(x, t)+\varepsilon F^{1}\left(x, \xi_{t}, t\right), \quad x(0)=x_{0}, \quad x \in R^{n}
$$

where $\xi_{t}$ represents a stationary stochastic process with zero mean, $F^{0}$ and $F^{1}$ are periodic in $t$ with period $T$. According to the stochastic averaging theorem, this process will converge weakly on a time interval of order $1 / \varepsilon^{2}$ to a diffusive Markov process $x^{0} t$ which is continuous almost surely and satisfies the Itô stochastic differential equation with the drift term $m_{i}(x)$ and diffusion matrix $\left(\sigma(x) \sigma^{T}(x)\right)$ given as

$$
\begin{aligned}
& m_{i}(x)=M_{t}\left\{F_{i}^{0}(x, t)+\int_{-\infty}^{0} E\left[\frac{\partial F_{i}^{1}}{\partial x_{j}}\left(x, \xi_{t}, t\right) F_{j}^{1}\left(x, \xi_{t+\tau}, t+\tau\right)\right] \mathrm{d} \tau\right\} \\
& \left(\sigma(x) \sigma^{T}(x)\right)=M_{t}\left\{\int_{-\infty}^{\infty} E\left[F_{k}^{1}\left(x, \xi_{t}, t\right) F_{j}^{1}\left(x, \xi_{t+\tau}, t+\tau\right)\right] \mathrm{d} \tau\right\}
\end{aligned}
$$

where

$$
M(\cdot)=\lim _{T \rightarrow \infty} \frac{1}{T} \int_{0}^{T}(\cdot) \mathrm{d} t
$$

and $E[\cdot]$ denotes the expectation.

\subsection{Deterministic Systems}

As a first step we perform the averaging by considering only the deterministic part (i.e., $\xi(t)=0$ ) of equation (20)

$$
\dot{A}=\varepsilon F_{1}(A, \phi) \quad \dot{\phi}=\Omega(A)+\varepsilon F_{2}(A, \phi) .
$$

By defining a near-identity transformation of the form

$$
\begin{aligned}
& A=\bar{A}+\varepsilon W_{1}(\bar{A}, \bar{\phi})+\varepsilon^{2} V_{1}(\bar{A}, \bar{\phi}) \\
& \phi=\bar{\phi}+\varepsilon W_{2}(\bar{A}, \bar{\phi})+\varepsilon^{2} V_{2}(\bar{A}, \bar{\phi})
\end{aligned}
$$

where $W_{i}$ and $V_{i}$ are first and second order transformations, respectively, and are taken to be functions of $(\bar{A}, \bar{\phi})$ which is different from the approach of Coppola [5]. In this paper, however, we only compute the averaged equations up to first order. The second order averaging will be necessary when the averaged of $F_{1}$ become zero in equation (21) (for example, when the deterministic perturbation in equation (8) has the form of $\varepsilon^{1 / 2} x^{n} \dot{x}^{m}, n$ : integer, $m$ : odd integer) as 
indicated in the Appendix. We can write down the averaged equations up to $O\left(\varepsilon^{2}\right)$ as

$$
\dot{\bar{A}}=\varepsilon \bar{F}_{1}+O\left(\varepsilon^{2}\right) \quad \dot{\bar{\phi}}=\bar{\Omega}+\varepsilon \bar{F}_{2}+O\left(\varepsilon^{2}\right)
$$

where $\bar{\Omega}=\Omega(\bar{A})$ and

$$
\bar{F}_{i}=\frac{1}{4 K} \int_{0}^{4 K} F_{i}(\bar{A}, u) \mathrm{d} u \text { and } u=4 K(k(\bar{A})) \bar{\phi} .
$$

The detailed derivation of equation (23), $W_{i}$ and $V_{i}$ can be found in the Appendix.

\subsection{Stochastic Systems}

We now consider equation (20) including the stochastic part. The set of Itô equations which include stochastic terms for the approximate amplitude $\bar{A}$ and phase $\bar{\phi}$ can be obtained as follows (we drop the overbar on $A$ and $\phi$ from now on for convenient notation)

$$
\begin{aligned}
& \mathrm{d} A=\varepsilon m_{A}(A) \mathrm{d} t+\varepsilon^{1 / 2} \sigma_{A} \mathrm{~d} w_{A} \\
& \mathrm{~d} \phi=\left[\bar{\Omega}+\varepsilon m_{\phi}(A)\right] \mathrm{d} t+\varepsilon^{1 / 2} \sigma_{\phi} \mathrm{d} w_{\phi}
\end{aligned}
$$

where

$$
\begin{aligned}
& m_{A}(A)=\bar{F}_{1}+M_{u}\left\{\int_{-\infty}^{0}\left\langle\frac{\partial G_{1}}{\partial A} G_{1, \tau}+\frac{\partial G_{1}}{\partial \phi} G_{2, \tau}\right\rangle R_{\xi \xi}(\tau) \mathrm{d} \tau\right\} \\
& m_{\phi}(A)=\bar{F}_{2}+M_{u}\left\{\int_{-\infty}^{0}\left\langle\frac{\partial G_{2}}{\partial A} G_{1, \tau}+\frac{\partial G_{2}}{\partial \phi} G_{2, \tau}\right\rangle R_{\xi \xi}(\tau) \mathrm{d} \tau\right\} \\
& {[\sigma \sigma]_{A A}=M_{u}\left\{\int_{-\infty}^{\infty}\left\langle G_{1} G_{1, \tau}\right\rangle R_{\xi \xi}(\tau) \mathrm{d} \tau\right\}} \\
& {[\sigma \sigma]_{A \phi}=M_{u}\left\{\int_{-\infty}^{\infty}\left\langle G_{1} G_{2, \tau}\right\rangle R_{\xi \xi}(\tau) \mathrm{d} \tau\right\}=0} \\
& {[\sigma \sigma]_{\phi \phi}=M_{u}\left\{\int_{-\infty}^{\infty}\left\langle G_{2} G_{2, \tau}\right\rangle R_{\xi \xi}(\tau) \mathrm{d} \tau\right\} .}
\end{aligned}
$$

$G_{i, \tau}(\cdot)=G_{i}(\cdot, t+\tau), R_{\xi \xi}(\tau)=E[\xi(t) \xi(t+\tau)], w_{A}$ and $w_{\phi}$ are independent standard Wiener process and the averaging operator is defined as

$$
M(\cdot)=\frac{1}{4 K} \int_{0}^{4 K}(\cdot) \mathrm{d} u \quad(u: \text { the argument of Jacobian elliptic function }) .
$$

Furthermore, the integrand in $[\sigma \sigma]_{A \phi}$ consists only of odd functions in $u$ for the case of linear parametric stochastic excitation. It is evident from $[\sigma \sigma]_{A \phi}=0$ the Itô equations of $A$ (equation (25)) and $\phi$ (equation (26)) are decoupled. Unlike the classical stochastic averaging method dealing with trigonometric functions in equation (20), it is difficult to find the closed form of the integral in equations (25) and (26) due to the presence of Jacobian elliptic functions with time shift. The following two approximate approaches can be used to obtain satisfactory results when $c_{1}$ and $c_{3}$ are positive constants. 
The first approximation is to expand each of the Jacobian elliptic functions ( $c n, d n, s n$ etc.) by its corresponding Fourier series, such as

$$
c n(u, k)=\frac{\pi}{k K} \sum_{n=0}^{\infty} \operatorname{sech}\left[\frac{(2 n+1) \pi K^{\prime}}{2 K}\right] \cos \left[\frac{(2 n+1) \pi u}{2 K}\right], \text { etc } .
$$

The second approximation follows from equation (10c), $0<k^{2}<0.5$ when $c_{1}$ and $c_{3}$ are positive constants. We can, thus, use the addition formulas of Jacobian elliptic functions, such as

$$
c n(u+v)=[c n u c n v+s n u s n v d n u d n v] /\left(1-k^{2} s n^{2} u s n^{2} v\right), \text { etc } .
$$

and Taylor expand those formulas up to a high order of $k^{2}$ about $k=0$ without expanding the elliptic functions themselves.

\section{Probability Density Function}

Since the Itô equation for amplitude $A$ (equation (25)) is uncoupled from the phase $\phi$ (equation (26)), the amplitude equation $A$ by itself constitutes a diffusive Markov process having a differential generator

$$
L(\cdot)=\varepsilon m_{A}(A) \frac{\partial(\cdot)}{\partial A}+\varepsilon \frac{1}{2}[\sigma \sigma]_{A A} \frac{\partial^{2}(\cdot)}{\partial A^{2}}
$$

Moreover, the transition probability density $p\left(A, t \mid A_{0}, t_{0}\right), t>t_{0}$ of the response process is governed by the Fokker-Planck equation

$$
\frac{\partial P(A, t)}{\partial t}=\frac{\partial}{\partial A}\left[m_{A} P(A, t)\right]+\frac{1}{2} \frac{\partial^{2}}{\partial A^{2}}\left[[\sigma \sigma]_{A A} P(A, t)\right]
$$

with initial condition $P\left(A, t \mid A_{0}, t_{0}\right) \rightarrow \delta\left(A-A_{0}\right)$ as $t \rightarrow t_{0}$. The stationary solution of equation (29) is

$$
P_{s t}(A)=\frac{N}{[\sigma \sigma]_{A A}} \operatorname{Exp}\left[\int^{A} \frac{2 m_{a}}{[\sigma \sigma]_{a a}} \mathrm{~d} a\right]
$$

where $N$ is the normalization constant. The solution of equation (30) can only be solved numerically due to the fact that the expression for the amplitude involves complicated combinations of Jacobian elliptic functions. Furthermore, the moment response can be obtained as

$$
E\left[A^{m}\right]=\int A^{m} P_{s t}(A) \mathrm{d} A
$$

\section{Examples}

Let us first consider the following nonlinear stochastic equation

$$
\ddot{x}+c_{1} x+c_{3} x^{3}+\varepsilon \beta \dot{x}+\varepsilon^{1 / 2} x \xi(t)=0
$$


where $c_{1} \geq 0, c_{3} \geq 0$ and $\beta$ is the damping coefficient. Equation (32) can be modeled as the equation of motion of beam subjected to stochastic excitation at the support.

In order to examine the instability regions of the trivial solution, we only have to study the linear problem, i.e. $c_{3}=0$ in equation (32). In this case and elliptic averaging is same as the trigonometric averaging, thus we first study equation (32) with $c_{3}=0$ by using the classical stochastic averaging method. The Itô equation for amplitude $A$, where $x=A \cos \phi$, can be obtained as

$$
\mathrm{d} A=\varepsilon\left(\frac{-\beta A}{2}+\frac{3 A S o}{16 c_{1}}\right) \mathrm{d} t+\varepsilon^{1 / 2}\left(\frac{S o}{8 c_{1}}\right) \mathrm{d} w_{A}
$$

and the stationary probability density function $P_{s t}$ can be given as

$$
P_{s t}(A)=N \frac{c_{1}}{S o} A^{\left(-8 \beta c_{1}+S o\right) / S o}
$$

where $S o$ is the spectral density of the white noise $\xi(t)$ and $N$ is the normalization constant. The almost sure stability of $A$ is characterized by the Lyapunov exponent $A<0$ which is obtained as

$$
\Lambda=\frac{-\beta}{2}+\frac{S o}{8 c_{1}} \text {. }
$$

The sample stability boundary of process $A$ of So v.s. $\beta$ is shown in Figure 1. We observe that in the stable region of Figure 1, the trivial solution $A=0$ is stable and thus expect the probability density function of $A$ to be a delta function. On the other hand, in the unstable region of Figure 1, the trivial solution $A=0$ loses its stability and the amplitude $A$ will increase without bound. However, in real physical system, the amplitude cannot become arbitrarily large. Thus, the corresponding nonlinear analysis as we derived in the previous sections is important in order to obtain the stationary amplitude densities.

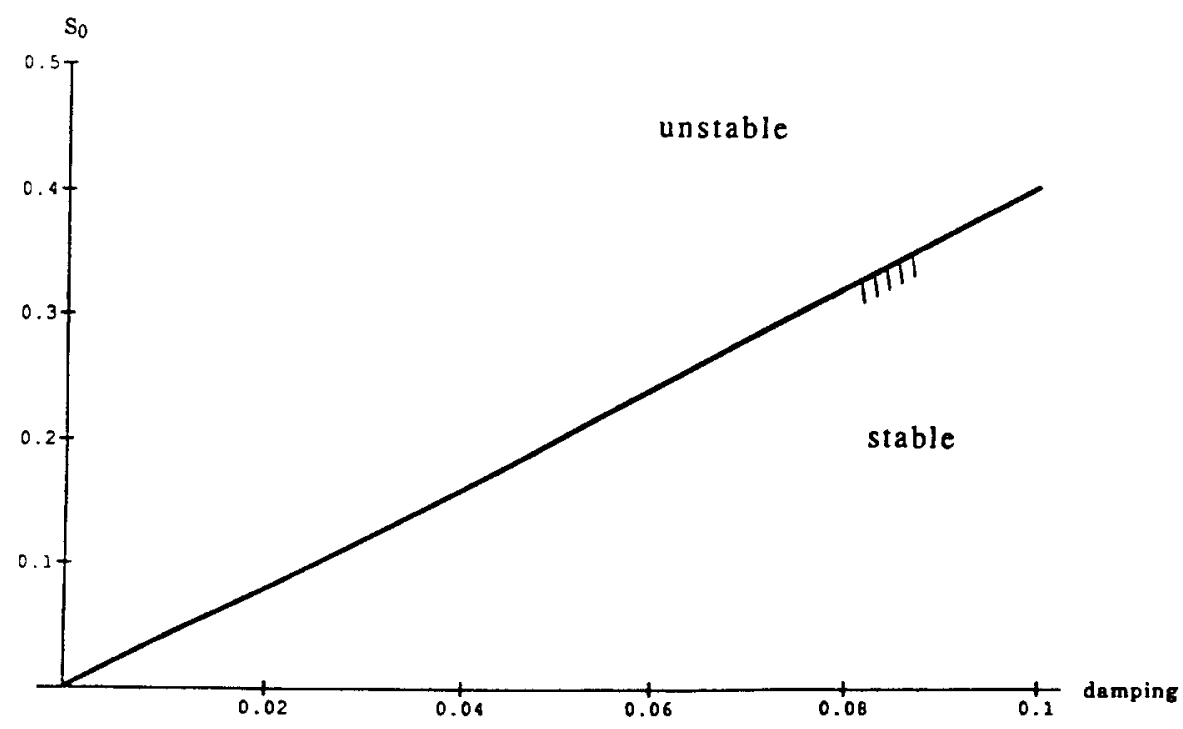

Fig. 1. Almost sure stability boundary. 
For the reasons stated above, we perform the nonlinear analysis by considering the cubic nonlinearity of equation (32) as the unperturbed term. The drift term and diffusion term of the Itô equation of amplitude $A$ (equations (23) and (26)) can be obtained as

$$
\begin{aligned}
m_{A}(A)= & M_{u}\left\{-\beta A d n^{2} s n^{2}\right\} \\
& +M_{u}\left\{\int_{-\infty}^{0}\left[\kappa_{1}\left(-c n^{2} d n^{2}+d n^{2} s n^{2}+k^{2} c n^{2} s n^{2}\right) k^{2} c n_{\tau}^{4}\right] R_{\xi \xi}(\tau) \mathrm{d} \tau\right\} \\
& +M_{u}\left\{\int_{-\infty}^{0}\left[\kappa_{2}\left(c n^{2} d n^{2}-d n^{2} s n^{2}-k^{2} c n^{2} s n^{2}\right) c n_{\tau}^{2}\right] R_{\xi \xi}(\tau) \mathrm{d} \tau\right\} \\
& +M_{u}\left\{\int_{-\infty}^{0}\left[\kappa_{3}\left(c n d n^{3} c n+k^{2} c n^{3} d n s n-k^{2} c n d n s n^{3}\right) c n_{\tau} d n_{\tau} s n_{\tau}\right] R_{\xi \xi}(\tau) \mathrm{d} \tau\right\} \\
& -M_{u}\left\{\int_{-\infty}^{0}\left[\left(\kappa_{1} k^{\prime 2}+\kappa_{3}\right) c n d n s n c n_{\tau} d n_{\tau} s n_{\tau}\right] R_{\xi \xi}(\tau) \mathrm{d} \tau\right\} \\
& +M_{u}\left\{\int_{-\infty}^{0}\left[\kappa_{3}\left(-c n^{2} d n^{2}+d n^{2} s n^{2}+k^{2} c n^{2} s n^{2}\right) z c n_{\tau} d n_{\tau} s n_{\tau}\right] R_{\xi \xi}(\tau) \mathrm{d} \tau\right\} \\
& +M_{u}\left\{\int_{-\infty}^{0}\left[\kappa_{1}\left(-c n^{2} d n^{2}+d n^{2} s n^{2}+k^{2} c n^{2} s n^{2}\right) z_{\tau} c n_{\tau} d n_{\tau} s n_{\tau}\right] R_{\xi \xi}(\tau) \mathrm{d} \tau\right\}
\end{aligned}
$$

where

$$
\begin{aligned}
& \kappa_{1}=\frac{A\left(2 k^{2}-1\right)}{k^{\prime 2} \omega^{2}}, \quad \kappa_{2}=\frac{A\left(2 k^{4}+k^{\prime 2}-k^{2}\right)}{k^{\prime 2} \omega^{2}}, \quad \kappa_{3}=\frac{A c_{1}}{k^{\prime 2} \omega^{4}}, k^{\prime 2}=1-k^{2} . \\
& {[\sigma \sigma]_{A A}=\underset{u}{M}\left\{\int_{-\infty}^{\infty}\left[\frac{A^{2}}{\omega^{2}} \text { cndnsn } c n_{\tau} d n_{\tau} s n_{\tau}\right] R_{\xi \xi}(\tau) \mathrm{d} \tau\right\} .}
\end{aligned}
$$

The subscript $\tau$ of the Jacobian elliptic functions represents the time shift from $t$ to $t+\tau$. The average equations of (36) and (37) thus, becomes

$$
m_{A}(A)=m_{A}(A)_{\text {deterministic }}+m_{A}(A)_{\text {stochastic }}
$$

where

$$
\begin{aligned}
m_{A}(A)_{\text {deterministic }}= & -\beta A\left(\frac{-1}{3}+\frac{1}{3 k^{2}}+\left(\frac{E}{K}\right)\left(\frac{2}{3}-\frac{1}{3 k^{2}}\right)\right) \\
m_{A}(A)_{\text {stochastic }}= & \kappa_{1} S o\left[\frac{-7}{30}-\frac{1}{10 k^{4}}+\frac{3}{10 k^{2}}+\frac{k^{2}}{30}+\left(\frac{E}{K}\right)^{2}\left(\frac{-1}{30}-\frac{1}{30 k^{4}}+\frac{1}{30 k^{2}}\right)\right. \\
& \left.+\frac{E}{K}\left(\frac{1}{10}+\frac{2}{15 k^{4}}-\frac{3}{10 k^{2}}-\frac{k^{2}}{15}\right)\right] \\
& +\kappa_{2} \operatorname{So}\left[\frac{-1}{15}-\frac{2}{15 k^{4}}+\frac{1}{5 k^{2}}+\frac{E}{K}\left(\frac{2}{15}+\frac{2}{15 k^{4}}-\frac{2}{15 k^{2}}\right)\right] \\
& +\kappa_{3} \operatorname{So}\left[\frac{1}{30}+\frac{1}{10 k^{4}}-\frac{1}{6 k^{2}}+\frac{k^{2}}{30}+\left(\frac{E}{K}\right)^{2}\left(\frac{-1}{30}-\frac{1}{30 k^{4}}+\frac{1}{30 k^{2}}\right)\right. \\
& \left.+\frac{E}{K}\left(\frac{1}{15}-\frac{1}{15 k^{4}}+\frac{1}{15 k^{2}}-\frac{k^{2}}{15}\right)\right]
\end{aligned}
$$




$$
[\sigma \sigma]_{A A}=\frac{A^{2}}{\omega^{2}} S o\left(\frac{-1}{15}-\frac{2}{15 k^{4}}+\frac{1}{5 k^{2}}+\frac{E}{K}\left(\frac{2}{15}+\frac{2}{15 k^{4}}-\frac{2}{15 k^{2}}\right)\right)
$$

where $K \equiv K(k)$ and $E \equiv E(k)$ are the complete elliptic integrals of the first and second kind, respectively. Furthermore, equations (38) and (39) are only dependent on amplitude $A$ through $k$, via (10c).

The stationary probability density function $P_{s t}$ and moment response then, can be obtained from equations (30) and (31), respectively. From the numerical calculation of $P_{s t}$, we can compare the linear results from Figure 1 and find that the unstable points $(\beta, S o)$ initially in Figure 1 can have probability densities and most probable value other than $A=0$ as shown in Figure 2 . Where the stable points $(\beta, S o)$ in Figure 1 correspond to the delta functions at $A=0$ and the unstable points $(\beta, S o)$ under the nonlinear analysis will have smooth density functions. Physically, as we mentioned before, this new elliptic averaging method give us more realistic results. The stationary density functions are plotted for different values of $S o$ in Figure 3.

The second example we would like to examine in a codimension two bifurcation system perturbed by multiplicative noise studied by Sri Namachchivaya [14]

$$
\dot{x}=y, \quad \dot{y}=-c_{1} x-x^{3}+\varepsilon\left[\left(c_{0}-\alpha x^{2}\right)\right] y+\varepsilon^{1 / 2} x \xi(t)
$$

where $c_{0}$ and $c_{1}$ are the unfolding parameters and $c_{0}=c_{1}=0$ defines the codimension two singularity point. In this example, we restrict our attention to the case in which $c_{0}<0$ and $c_{1}>0$ and reverse the analysis of the remaining cases for a later paper.

The drift term and diffusion term of the Itô equation of amplitude $A$, where $A=x / c n(u, k)$, are similar to equations (38) and (39) respectively. However, there is one additional drift term

$$
\underset{u}{M}\left\{-\alpha A^{3} c n^{2} d n^{2} s n^{2}\right\}=-\alpha A^{3}\left(\frac{-1}{15}+\frac{1}{5 k^{2}}-\frac{2}{15 k^{4}}+\left(\frac{E}{K}\right)\left(\frac{2}{15}-\frac{2}{15 k^{2}}-\frac{2}{15 k^{4}}\right)\right)
$$

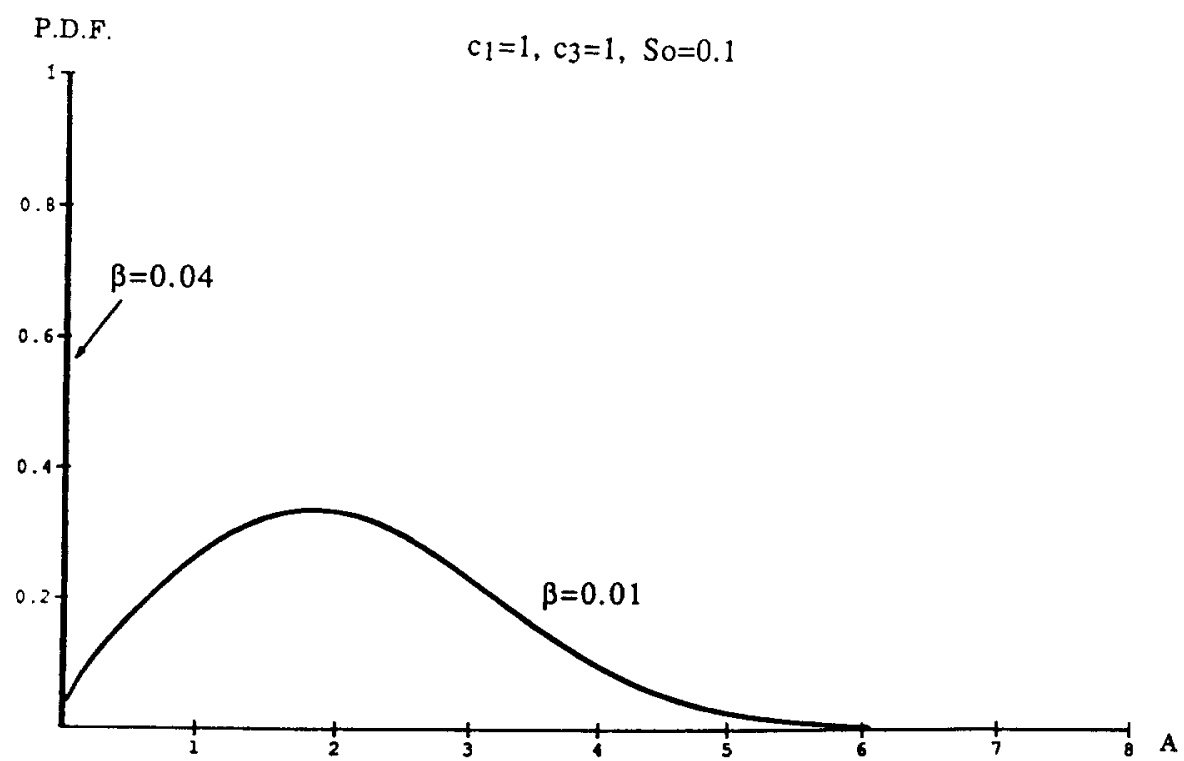

Fig. 2. Probability density function of amplitude $A$. 


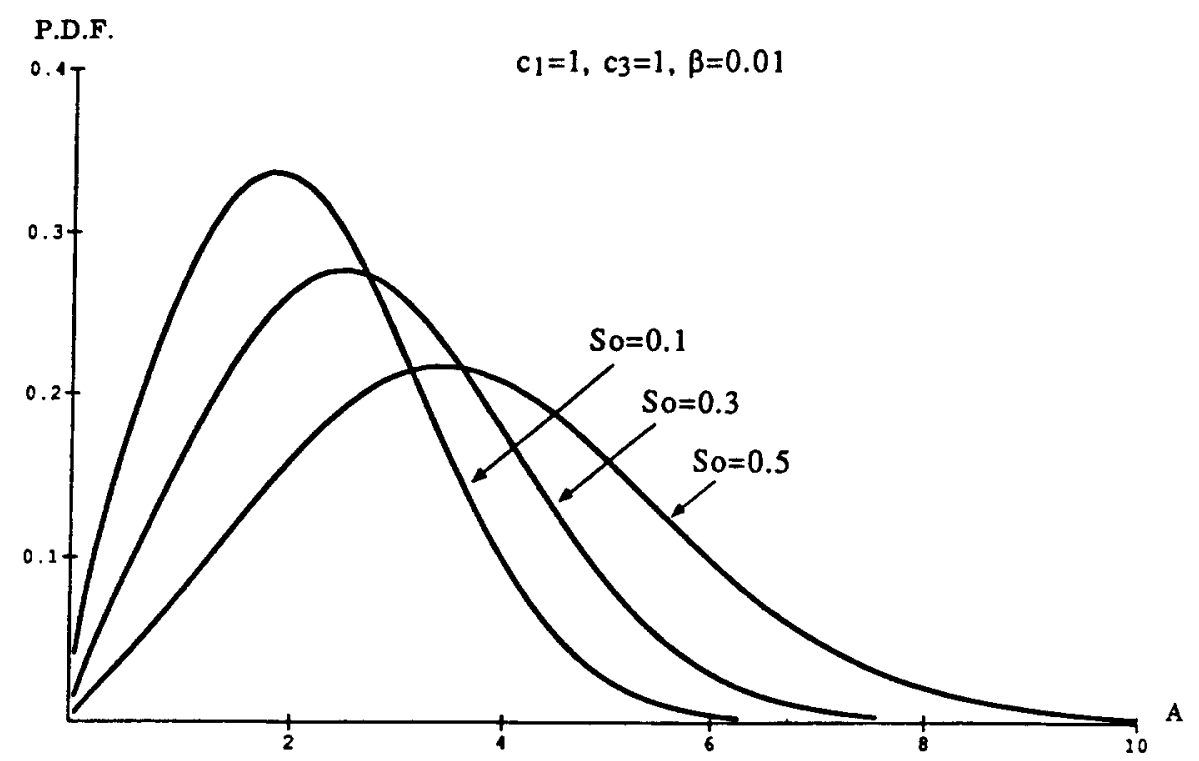

Fig. 3. Variation of density function with $S_{0}$.

from the deterministic term $\alpha x^{2} y$ of equation (40). That is

$$
\begin{aligned}
m_{A}(A)_{\text {deterministic }}= & -c_{0} A\left(\frac{-1}{3}+\frac{1}{3 k^{2}}+\left(\frac{E}{K}\right)\left(\frac{2}{3}-\frac{1}{3 k^{2}}\right)\right) \\
& -\alpha A^{3}\left(\frac{-1}{15}+\frac{1}{5 k^{2}}-\frac{2}{15 k^{4}}+\left(\frac{E}{K}\right)\left(\frac{2}{15}-\frac{2}{15 k^{2}}-\frac{2}{15 k^{4}}\right)\right)
\end{aligned}
$$

$m_{A}(A)=m_{A}(A)_{\text {deterministic }}[$ in (41a) $]+m_{A}(A)_{\text {stochastic }}[$ in (38b) $]$.

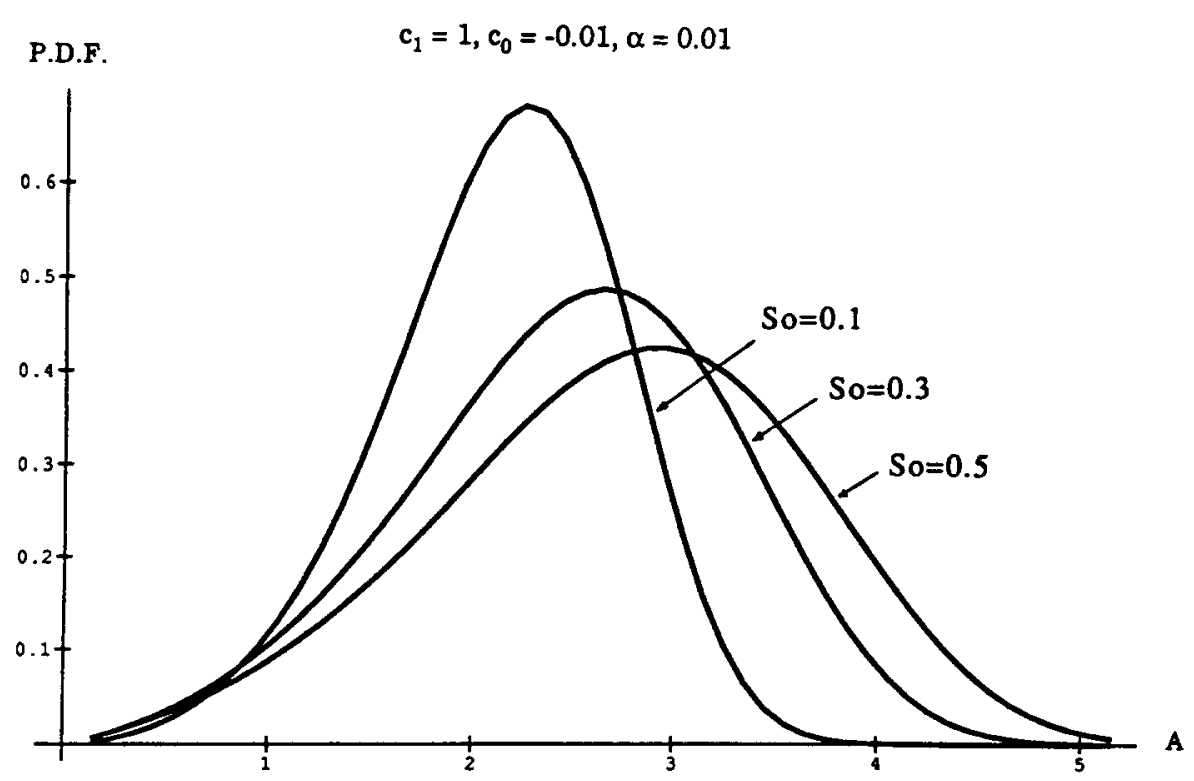

Fig. 4. Variation of density function with $S_{0}$. 
A stable limit cycle of amplitude $A=1.8673$ can be found by numerically solving $m_{A}(A)=0$ in equation (41b) for deterministic system at $c_{1}=1, c_{0}=-0.01$ and $\alpha=0.01$. The parametric noise to the system will have a shift effect from the most probable value point of view as shown in Figure 4, where the stationary density functions are plotted for different values of So.

\section{Conclusion}

In this paper, we have developed the method of stochastic averaging by using elliptic functions. Unlike the "regular" trigonometric stochastic averaging, this method considers the strong (cubic) nonlinearity as a part of the unperturbed equation and uses elliptic functions to express the averaged equations. We also provided some examples and demonstrated the importance of the nonlinear terms in the stochastic systems. The elliptic averaging methods retains those terms in the response equations. Moreover, by using these response equations, we can describe the behavior of nonlinear stochastic systems in a more realistic manner. However, the extension of this method for multi-degree-of-freedom systems will be cumbersome even with the use of symbolic manipulator such as Mathematica, Macsyma and Maple.

\section{Acknowledgements}

This research was partially supported by the Air Force Office of Scientific Research through Grant 91-0041 monitored by Dr. Spencer Wu and V. Coppola was supported by the ONT Postdoctoral Fellowship Program.

\section{Appendix: Derivation of Deterministic Averaging}

The following derivation is slightly different from that contained in Coppola [5] but equivalent to first order. We would like to obtain the averaged equations corresponding to equation (21) as

$$
\begin{aligned}
& \dot{\bar{A}}=\varepsilon Q_{1}(\bar{A}, \bar{\phi})+\varepsilon^{2} Q_{2}(\bar{A}, \bar{\phi})+O\left(\varepsilon^{3}\right) \\
& \dot{\bar{\phi}}=\Omega(\bar{A})+\varepsilon R_{1}(\bar{A}, \bar{\phi})+\varepsilon^{2} R_{2}(\bar{A}, \bar{\phi})+O\left(\varepsilon^{3}\right) .
\end{aligned}
$$

Differentiating equation (22) and substituting terms from equation (A1), we obtain the following equation in $(\dot{A}, \dot{\phi})$

$$
\begin{aligned}
& \dot{A}=\varepsilon\left(Q_{1}+\bar{\Omega} W_{1 \phi}\right)+\varepsilon^{2}\left(Q_{2}+Q_{1} W_{1 A}+R_{1} W_{1 \phi}+V_{1 \phi} \bar{\Omega}\right)+O\left(\varepsilon^{3}\right) \\
& \dot{\phi}=\bar{\Omega}+\varepsilon\left(R_{1}+\bar{\Omega} W_{2 \phi}\right)+\varepsilon^{2}\left(R_{2}+Q_{1} W_{2 A}+R_{1} W_{2 \phi}+V_{2 \phi} \bar{\Omega}\right)+O\left(\varepsilon^{3}\right)
\end{aligned}
$$

where we let $\Omega(\bar{A})=\bar{\Omega}, \partial W_{1} / \partial \bar{A}=W_{1 A}, \partial W_{1} / \partial \bar{\phi}=W_{1 \phi}$, etc. We now expand $F_{1}, F_{2}$ and $\Omega$ in a power series in $\varepsilon$ : 


$$
\begin{aligned}
& F_{1}(A, \phi)=F_{1}(\bar{A}, \bar{\phi})+\varepsilon W_{1} F_{1 A}(\bar{A}, \bar{\phi})+\varepsilon W_{2} F_{1 \phi}(\bar{A}, \bar{\phi})+O\left(\varepsilon^{2}\right) \\
& F_{2}(A, \phi)=F_{2}(\bar{A}, \bar{\phi})+\varepsilon W_{1} F_{2 A}(\bar{A}, \bar{\phi})+\varepsilon W_{2} F_{2 \phi}(\bar{A}, \bar{\phi})+O\left(\varepsilon^{2}\right) \\
& \Omega(A)=\bar{\Omega}+\varepsilon \bar{\Omega}_{A} W_{1}+\varepsilon^{2} \bar{\Omega}_{A} V_{1}+\frac{1}{2} \varepsilon^{2} \bar{\Omega}_{A A} W_{1}^{2}+O\left(\varepsilon^{3}\right) .
\end{aligned}
$$

Substituting equation (A3) into equation (21) and comparing this with equation (A2) we obtain the following equations

$\varepsilon$ terms:

$$
\begin{aligned}
& F_{1}(\bar{A}, \bar{\phi})=Q_{1}(\bar{A})+\bar{\Omega}(\bar{A}) W_{1 \phi}(\bar{A}, \bar{\phi}) \\
& F_{2}(\bar{A}, \bar{\phi})+\bar{\Omega}(\bar{A})_{A} W_{1}(\bar{A}, \bar{\phi})=R_{1}(\bar{A})+\bar{\Omega}(\bar{A}) W_{2 \phi}(\bar{A}, \bar{\phi})
\end{aligned}
$$

\section{$\varepsilon^{2}$ terms:}

$$
\begin{aligned}
& W_{1}(\bar{A}, \bar{\phi}) F_{1 A}(\bar{A}, \bar{\phi})+W_{2}(\bar{A}, \bar{\phi}) F_{1 \phi}(\bar{A}, \bar{\phi}) \\
& \quad=Q_{2}(\bar{A})+Q_{1}(\bar{A}) W_{1 A}(\bar{A}, \bar{\phi})+R_{1}(\bar{A}) W_{1 \phi}(\bar{A}, \bar{\phi})+V_{1 \phi}(\bar{A}, \bar{\phi}) \bar{\Omega}(\bar{A}) \\
& \bar{\Omega}_{A}(\bar{A}) V_{1}(\bar{A}, \bar{\phi})+\frac{1}{2} \bar{\Omega}_{A A}(\bar{A}) W_{1}^{2}(\bar{A}, \bar{\phi})+W_{1}(\bar{A}, \bar{\phi}) F_{2 A}(\bar{A}, \bar{\phi})+W_{2}(\bar{A}, \bar{\phi}) F_{2 \phi}(\bar{A}, \bar{\phi}) \\
& \quad=R_{2}(\bar{A})+Q_{1}(\bar{A}) W_{2 A}(\bar{A}, \bar{\phi})+R_{1}(\bar{A}) W_{2 \phi}(\bar{A}, \bar{\phi})+V_{2 \phi}(\bar{A}, \bar{\phi}) \bar{\Omega}(\bar{A})
\end{aligned}
$$

Substituting equations (A4) and (A5) for $Q_{1}, Q_{2}, R_{1}$ and $R_{2}$ into equation (A1) we obtain the following equations in $(\bar{A}, \phi)$

$$
\begin{aligned}
& \dot{\bar{A}}=\varepsilon\left[F_{1}(\bar{A}, \bar{\phi})-\bar{\Omega} W_{1 \phi}\right]+\varepsilon^{2}\left[H_{1}(\bar{A}, \bar{\phi})-V_{1 \phi} \bar{\Omega}\right]+O\left(\varepsilon^{3}\right) \\
& \dot{\bar{\phi}}=\bar{\Omega}+\varepsilon\left[F_{2}(\bar{A}, \bar{\phi})+\bar{\Omega}_{A} W_{1}-\bar{\Omega} W_{2 \phi}\right]+\varepsilon^{2}\left[H_{2}(\bar{A}, \bar{\phi})-V_{2 \phi} \bar{\Omega}\right]+O\left(\varepsilon^{3}\right)
\end{aligned}
$$

where

$$
\begin{aligned}
& H_{1}(\bar{A}, \bar{\phi})=W_{1} F_{1 A}+W_{2} F_{1 \phi}-Q_{1} W_{1 A}-R_{1} W_{1 \phi} \\
& H_{2}(\bar{A}, \bar{\phi})=\bar{\Omega}_{A} V_{1}+\frac{1}{2} \bar{\Omega}_{A A} W_{1}^{2}+W_{1} F_{2 A}+W_{2} F_{2 \phi}-Q_{1} W_{2 A}-R_{1} W_{2 \phi}
\end{aligned}
$$

$W_{i \phi}$ and $V_{i \phi}$ in (A4) and (A5), respectively, must satisfy in order for equations (22) and (A1) to hold.

Since each $F_{i}$ is periodic, $F_{i}$ can be considered as the sum of its mean value $\bar{F}_{i}(\bar{A})$ and an oscillating part with zero mean $\left(\tilde{F}_{i}\right)$ where

$$
\bar{F}_{i}=\frac{1}{4 K} \int_{0}^{4 K} F_{i}(\bar{A}, \bar{u}) \mathrm{d} \bar{u} \text { and } \bar{u}=4 K(k(\bar{A})) \bar{\phi} .
$$

Upon requiring equation (22) be a valid asymptotic series for all time, the natural choice for $W_{i}$ and $V_{i}$ in (A4) and (A5) are to require $W_{i \phi}$ and $V_{i \phi}$ to be periodic with zero mean so that $W_{i}$ and $V_{i}$ themselves are periodic and bounded. This leads to the choices: 


$$
\begin{aligned}
& Q_{1}=\bar{F}_{1} \\
& \bar{\Omega} W_{1 \phi}=\tilde{F}_{1}=F_{1}-\bar{F}_{1} \\
& W_{1}=\frac{1}{\bar{\Omega}} \int W_{1 \phi} \mathrm{d} \phi=\frac{1}{\omega} \int\left(F_{1}(\bar{A}, \bar{u})-\bar{F}_{1}\right) \mathrm{d} u+W_{10}
\end{aligned}
$$

and

$$
\begin{aligned}
& R_{1}=\bar{F}_{2} \\
& \bar{\Omega} W_{2 \phi}=\tilde{F}_{2}+\bar{\Omega}_{A} W_{1}=F_{2}-\bar{F}_{2}+\bar{\Omega}_{A} W_{1} \\
& W_{2}=\frac{1}{\bar{\Omega}} \int W_{2 \phi} \mathrm{d} \phi=\frac{1}{\omega} \int\left(F_{2}(\bar{A}, \bar{u})-\bar{F}_{2}+\bar{\Omega}_{A} W_{1}\right) \mathrm{d} \bar{u}+W_{20}
\end{aligned}
$$

where the integration constants $W_{i 0}$ have been chosen so that $W_{i}$ have zero mean. This completes the first order averaging. Furthermore, equation (A6) implies

$$
V_{i}=\frac{1}{\omega} \int\left(H_{i}(\bar{A}, \bar{u})-\bar{H}_{i}\right) \mathrm{d} \bar{u}+V_{i 0}
$$

where, again, the integration constant $V_{i 0}$ is chosen such that $V_{i}$ has zero mean. Finally, from equations (A4), (A5), (A9) and (A10) the averaged equations can be written as equation (23).

\section{References}

1. Ariaratnam, S. T. and Srikantaiah., T. K., 'Parametric instabilities in elastic structure', J. Stru. Mech. 8, 1978, 349-365.

2. Borodin, A. N., 'A limit theory for solutions of differential equation with random right-hand side', Theory Probab. Appl. 2, 1977, 575-595.

3. Byrd, P. and Friedman, M., Handbook of Elliptic Integrals for Engineering and Scientists, Springer-Verlag, Berlin, 1954.

4. Coppola, V. T. and Rand, R. H., 'Averaging using elliptic functions: approximation of limit cycles', Acta Mechanica 81, 1990, 125-142.

5. Coppolar, V. T., Averaging of strongly nonlinear oscillators using elliptic functions, PhD. Dissertation, Cornell University, Ithaca NY, 1989.

6. Khasminskii, R. Z., 'On stochastic processes defined by differential equations with a small parameter', Theory Probab. Appl. 11, 1966, 211-228.

7. Khasminskii, R. Z., 'On the principal of averaging for Itô stochastic differential equation', Kybernetika (Prague) 4, $1968,260-279$.

8. Lin, Y. K., 'Some observations of the stochastic averaging method', Proc. Eng. Mech. 1, 1986, $23-27$.

9. Papanicolaou, G. C. and Kohler, W., 'Asymptotic analysis of deterministic and stochastic equations with rapidly varying components', Commun. Math. Phys. 46, 1976, 217-232.

10. Papanicolaou, G. C., Stroock, D., and Varadhan, S. R. S., 'Martingale approach to some limits theorems', in The Duke Turbulence Conference (David Ruelle, ed.), 1976.

11. Roberts, J. B. and Spanos, P. D., 'Stochastic averaging: an approximate method of solving random vibration problems', Int. J. Non-Linear Mech. 21, 1986, 111-134.

12. Roberts, J. B., 'The energy envelope of randomly excited non-linear oscillator', J. Sound Vib. 60, 1978, $177-185$.

13. Sri Namachchivaya, N. and Ariaratnam, S. T., 'Stochastically perturbed linear gyroscopic systems', Mech. Struct. \& Mach 15, 1987, 323-345.

14. Sri Namachchivaya, N., 'Co-dimension two bifurcations in the presence of noise', J. Appl. Mech. 58, 1990, $259-265$.

15. Stratonovich, R. L., Topics in the Theory of Random Noise, Vol. 1, Gordon and Breach, New York, 1963. 